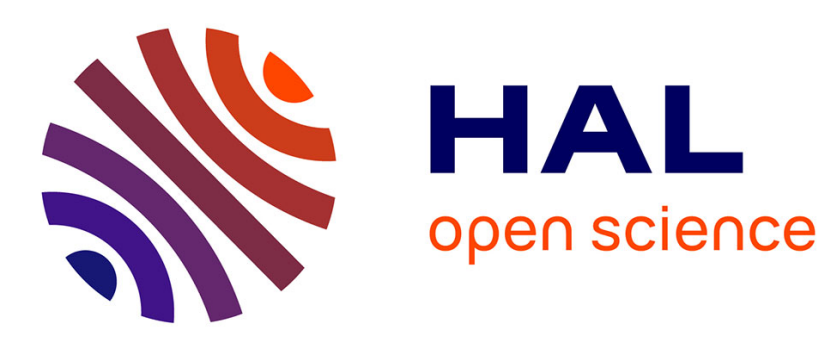

\title{
Haptic Communication in Collaborative Virtual Environments
}

\author{
Jinling Wang, Amine Chellali, Caroline G. L. Cao
}

\section{To cite this version:}

Jinling Wang, Amine Chellali, Caroline G. L. Cao. Haptic Communication in Collaborative Virtual Environments. Human Factors, 2016, 58 (3), pp.496-508. 10.1177/0018720815618808 . hal01223967

\section{HAL Id: hal-01223967 \\ https://hal.science/hal-01223967}

Submitted on 8 Jan 2016

HAL is a multi-disciplinary open access archive for the deposit and dissemination of scientific research documents, whether they are published or not. The documents may come from teaching and research institutions in France or abroad, or from public or private research centers.
L'archive ouverte pluridisciplinaire HAL, est destinée au dépôt et à la diffusion de documents scientifiques de niveau recherche, publiés ou non, émanant des établissements d'enseignement et de recherche français ou étrangers, des laboratoires publics ou privés. 


\title{
Haptic Communication in Collaborative Virtual Environments
}

\author{
Jinling Wang \\ Wright State University, Dayton, OH USA \\ Amine Chellali \\ Universite d'Evry Val d'Essonne, Evry, France \\ Caroline GL Cao \\ Wright State University, Dayton, OH USA
}

\author{
Running head: HAPTIC COMMUNICATION IN COLLABORATION \\ Manuscript type: Research Article
}

Exact word count of text: 4,490

Corresponding author:

Caroline GL Cao, caroline.cao@wright.edu, 937-775-5044

Department of Biomedical, Industrial \& Human Factors Engineering, Wright State University, 3640 Colonel Glenn Hwy, Dayton, OH 45435, USA

\section{Acknowledgements:}

This work was supported in part by a grant from the NIH (NIBIB 1R01EB009362-01A2) and an award from the Ohio Third Frontier to the Ohio Imaging Research and Innovation Network (OIRAIN). 


\begin{abstract}
Objective: To understand the interaction between haptic and verbal communication, this project quantified the relative effect of verbal, haptic, and haptic+verbal feedback in a collaborative virtual pointing task.

Background: Collaborative Virtual Environments (CVEs) provide a medium for interaction among remote participants. Better understanding of the role of haptic feedback as a supplement to verbalization can improve the design of CVEs.
\end{abstract}

Methods: Thirty-six participants were randomly paired into 18 dyads to complete a 2D pointing task in a CVE. In a mixed experimental design, participants completed the task in three communication conditions: haptic only $(\mathrm{H})$, verbal only $(\mathrm{V})$, and haptic+verbal (HV). The order of the conditions presented to the participants was counter-balanced.

Results: The time to task completion, path length, overshoot, and RMSE were analyzed. Overall, performance in the $\mathrm{V}$ and $\mathrm{HV}$ conditions were significantly better than in the $\mathrm{H}$ condition. Haptics only was the least efficient communication channel, but elicited response with the shortest reaction time. When verbalization was not available, the use of the haptic device was more likely to be exaggerated to ensure information transmission. When verbalization was used, participants converged on the use of Cartesian coordinate system for communicating spatial information.

Conclusion: Haptic communication can be used to complete a collaborative virtual task, but is less efficient than verbal communication. A training period may help to improve the efficiency of haptic communication.

Application: These results can be used to design remote collaboration tasks incorporating haptic components, and for improving the design of CVEs that support haptic communication. 
Keywords: computer-supported collaborations, team communication, multimodality, virtual environments, team collaboration

Précis: Better understanding the role of haptic feedback as a supplement to verbalization can help to improve the design of Collaborative Virtual Environments. This project studies the utility of verbal feedback, haptic feedback, and haptic+verbal feedback in a collaborative virtual pointing task. 


\section{INTRODUCTION}

Collaborative Virtual Environments (CVEs) allow multiple participants located remotely to interact and collaborate in a virtual space (Churchill, Snowdon, \& Munro, 2001). By sharing the same virtual space, team members can work together from anywhere in the world. CVEs are expected to save time, improve efficiency, and increase productivity. Applications for these systems can be found in medicine (e.g., telemedicine), the design industry (e.g., computer aided design and assembly), and entertainment (e.g., internet gaming) (Hanna \& Richards, 2014; Churchill \& Snowdon, 1998; Young, Moon, Sohn, \& Fernandes, 2008). (See Churchill \& Snowdon, 1998 and Wright \& Madey, 2008 for detailed reviews of state-of-the-art technology in CVE).

In general, CVE systems offer new interaction possibilities by allowing users to share virtual workspaces and communicate remotely using new communication modalities. However, there is a lack of guidelines for designing VE that support specific collaborative tasks. In the real world, operators organize their behaviors according to their environment (such as knowledge of their physical surrounding, position of their partner and other shared objects, and their sense of copresence in the same environment) to improve their collaboration (Harrison \& Dourish, 1996; Chellali, Milleville-Pennel, \& Dumans, 2013). In CVEs, some of these physical features become inaccessible (Chellali, Dumas, \& Milleville-Pennel, 2011). For instance, spatial relationships amongst actors and objects in the CVE are easily assessed through vision and sound. But physical properties of objects in the CVE such as softness or stiffness are not available without haptic feedback. This may affect the grounding process and constrain the collaborative activities. It is therefore important to investigate the different interaction and communication possibilities offered by the haptic modality. 


\section{Haptic communication in real and virtual environments}

In the physical world, haptic communication is very informative. A common scenario can be found during furniture handling, such as when two people are carrying a table through a fully furnished room, and one of them is advancing while the other is walking backwards. The person who is walking backwards may be verbally instructed by the forward-walking individual while simultaneously feeling the push and pull of the speaker through the table. Alternatively, if both furniture handlers were facing the same direction, the leading individual would have a better view of the path ahead, such as an obstacle or a narrow doorway, thus requiring a side step to the left or right, or tilting the table slightly to deliberately signal a change of position. Clearly, haptic communication is being used here. Haptics, unlike the visual and auditory modalities, can provide a very direct and intuitive physical connection between team members in a virtual space (Chellali et al., 2011). It can allow users to feel the interactions of manipulation and enhance the sense of presence (Burdea, 1996). Shared haptic feedback, such as force, position, and motion information, between remote users while manipulating virtual objects, can thus enhance their mutual understanding of a shared task to achieve a common goal during collaboration.

Communication in CVEs is more difficult compared with a face-to-face communication. While various verbal and non-verbal cues are used to communicate and establish common ground (mutual understanding of a shared situation) (Clark \& Brennan, 1991) when working face-toface, remote communication is usually devoid of non-verbal cues. This disrupts the grounding process that is necessary to efficiently complete a collaborative task (Clark \& Brennan, 1991), and can lead to misunderstandings (Chellali et al., 2013; Gergle, Kraut, \& Fussell, 2012).

Studies on haptic communication and collaboration in virtual environments have been carried out in various applications. For instance, Gunn (2006) developed a system in which two remote 
partners can work together on a virtual sculpture, each using a separate haptic device. However, there was no direct haptic interaction between partners even though haptic information is critical in the sculpting task. Other researchers have investigated the role of haptics during comanipulation of virtual objects (Basdogan, Ho, Srinivasan, \& Slater, 2000; Buxton, 1995; Chellali et al., 2011) using two haptic devices. All showed that haptic interaction enhanced the collaborative performance. While haptic communication was clearly used by partners during these co-manipulation tasks, the role of haptics in communication was not investigated. On the other hand, Chellali, Dumas, \& Milleville-Pennel (2012) investigated the role of haptic communication to support medical simulation and training by allowing an expert surgeon to guide a trainee's hand while performing a surgical task. They concluded that haptic communication could help an expert to transfer his knowledge to novices. The same guidance metaphor was used by Ullah, Liu, Otmane, Richard, \& Mallem (2011) for teaching handwriting. These authors also concluded that haptic communication was helpful for teaching handwriting, but only subjective measurements were taken. More generally, research has shown that haptic feedback can make a positive contribution by increasing the degree of mutual awareness (García, Molina, González, Martínez, \& Martínez, 2009; Hubbold, 2002), enhancing the feeling of copresence with remote partners and confidence during their collaboration (Basdogan et al., 2000; Chellali et al., 2011).

To improve the design of CVEs and enhance collaboration, the use of different interaction modalities in combination have been suggested, such as 3D stereoscopic vision, spatial sounds, and haptic feedback (Moll, Huang, \& Sallnas, 2010; Lu, Liu, \& Liu, 2012; U1lah, 2011). Sensory modalities can also substitute for others that are unavailable or difficult to implement. For instance, when haptic information is missing, the visual channel can extract force information 
through "visual force feedback" (e.g., inferring how much force has been applied through the amount of surface indentation or object deflection caused by the interaction). This has been referred to as "pseudo haptic feedback" and is based on proprieties of human visuo-haptic perception (Lecuyer, 2009). However, the use of the haptic channel to supplement or support other modalities, such as verbal communication, in CVEs has not been extensively investigated. Studies on the combination of haptics and visual feedback in virtual environments show improved task performance compared with visual feedback alone (Basdogan et al., 2000; Lu et al., 2012). Huang, Moll, Sallnäs, and Sundblad (2012) compared task performance in the audio+haptic+visual feedback condition with that in the haptic+visual feedback condition. In their study, audio feedback was used to inform the partner about one's actions and location in the virtual environment. Their results show that the audio+haptic+visual feedback condition was more efficient. The role of negotiation and dominance in haptic collaboration has also been studied (Reed \& Peshkin, 2008). The mutual haptic feedback had a more stable effect on the dominance behavior across time. However, less attention has been paid to understanding how people use the haptic modality to convey and exchange information in CVEs with/without other modalities and the combination of this modality with verbal communication. For instance, while verbal feedback is the primary modality for information exchange in collaborative settings, the role of haptic feedback as a supplement to verbalization in team communication and the combination of both channels is still an open research question.

The long term goal of our research is to understand the interaction between haptic and verbal feedback and to investigate people's ability to communicate through haptics. Our preliminary studies have focused on the role of haptic interaction as a communication channel for a target pointing task (Takac et al., 2011; Wang, Chellali, \& Cao, 2013). These studies show that haptic 
communication is used to correctly complete the task and suggest that several communication strategies are developed depending on whether haptic and/or verbal communication channels are used (Takac et al., 2011; Wang et al., 2013). In this study, we investigate the relative utility of haptic, verbal, and haptic+verbal feedback in a collaborative virtual pointing task. The hypothesis was that haptic feedback would improve performance and enhance communication effectiveness in a remote collaborative virtual task. This research is a step towards a deeper understanding of how the haptic channel is used in remote collaboration so as to improve the design of CVEs that support haptic communication.

\section{METHODS}

\section{Participants}

A total of 36 participants ( 21 male, 15 female; aged 18-70, mean=25, $\mathrm{SD}=11.6$ ) with no previous experience with haptic devices were recruited. Twenty-four of them were native English speakers while the others spoke English as a second language. Thirty-two of them were righthanded, three were left-handed, and one was ambidextrous. The participants were randomly paired into 18 dyads, each containing an Acting Agent and a Supervisor. The participant who sat first in the chair designated for the Acting Agent was assigned the role of Acting Agent while the other participant was assigned the role of Supervisor. Participants switched roles midpoint during the experiment so that each experienced both roles.

\section{Task and Procedure}

In this study, a pointing task in a 2D virtual environment was used. Each participant of the dyad sat in front of a 23" monitor. The Acting Agent was required to point to an invisible target on his/her computer monitor, using a mouse as the pointing device, when directed by the Supervisor who could see the target on his/her own computer monitor. Participants were instructed to 
complete the task collaboratively as quickly as possible. The Supervisor could see the starting point (yellow square in the center of the monitor), the cursor (red square), and the target (green square) on the monitor, as shown in Figure 1, but could not control the motion of the cursor. On the other hand, the Acting Agent could use the mouse held in his/her dominant hand to move the cursor, but could not see the green target. A curtain was drawn between the two participants so that they could not observe their partner's monitor screen or body language. A cardboard box was used to block the haptic device from the Acting Agent's view.

To complete the task, the participants communicated with each other using either verbal communication only, haptic communication only, or a combination of verbal and haptic communication. For verbal communication, the participants were allowed to talk to each other in whatever manner they wished, conveying whatever information they wished, without restriction. The task was for the Supervisor to provide verbal commands to guide the Acting Agent to move the mouse to an invisible target on the Acting Agent's computer monitor. Haptic communication was achieved through the use of two linked haptic devices (PHANToM Omni, SensAble Technologies). This haptic device could provide 6 degree-of-freedom (DOF) positional sensing and 3 DOF force feedback. The two haptic devices were connected in a master-slave fashion. As shown in Figure 1, the supervisor held the master device using his/her dominant hand while the Acting Agent held the slave device with the non-dominant hand. The haptic devices were linked using the position control method so that the master arm could remotely move the slave arm on the three translation axes. The Supervisor could move the stylus of the master haptic device freely. Meanwhile, the stylus of the slave haptic device held by Acting Agent would mimic the movement of the Supervisor's stylus. The Acting Agent could feel the motion of the Supervisor through the slave haptic device (such as direction, distance, and speed of motion) but could not 
control the stylus of this device. Therefore, in the haptic communication only condition, the Supervisor must guide the Acting Agent by physically moving the haptic device to indicate the target location relative to current cursor position, without talking. In the verbal+haptic communication condition, the Supervisor could provide verbal commands to the Acting Agent as well as use the haptic device to direct the Acting Agent.

Before beginning the experiment, the participants were allowed 2 minutes to observe both monitor screens simultaneously and experience the haptic link to become familiar with the experimental setup and the task, such as the master-slave linkage of the haptic devices.

In each experimental condition, a total of 32 targets were generated and presented randomly to the participants (16 before the dyads switched roles, and 16 after). The 16 targets were at different distances from the starting position, resulting in 4 different indices of difficulty ( 4 target locations were randomly generated for each level). According to Shannon's formulation of Fitts' Law (MacKenzie, 1992), the Index of Difficulty (ID) is defined as follows:

$$
\mathrm{ID}=\log _{2}\left(1+\frac{D}{W}\right)
$$

where $D$ is the distance between the starting position and the target location, $W$ is the width of the target measured along the axis of motion. The size of the target used in this study was $10 \times$ 10 pixels. The four IDs were 2.0, 2.5, 3.0, and 3.5. 


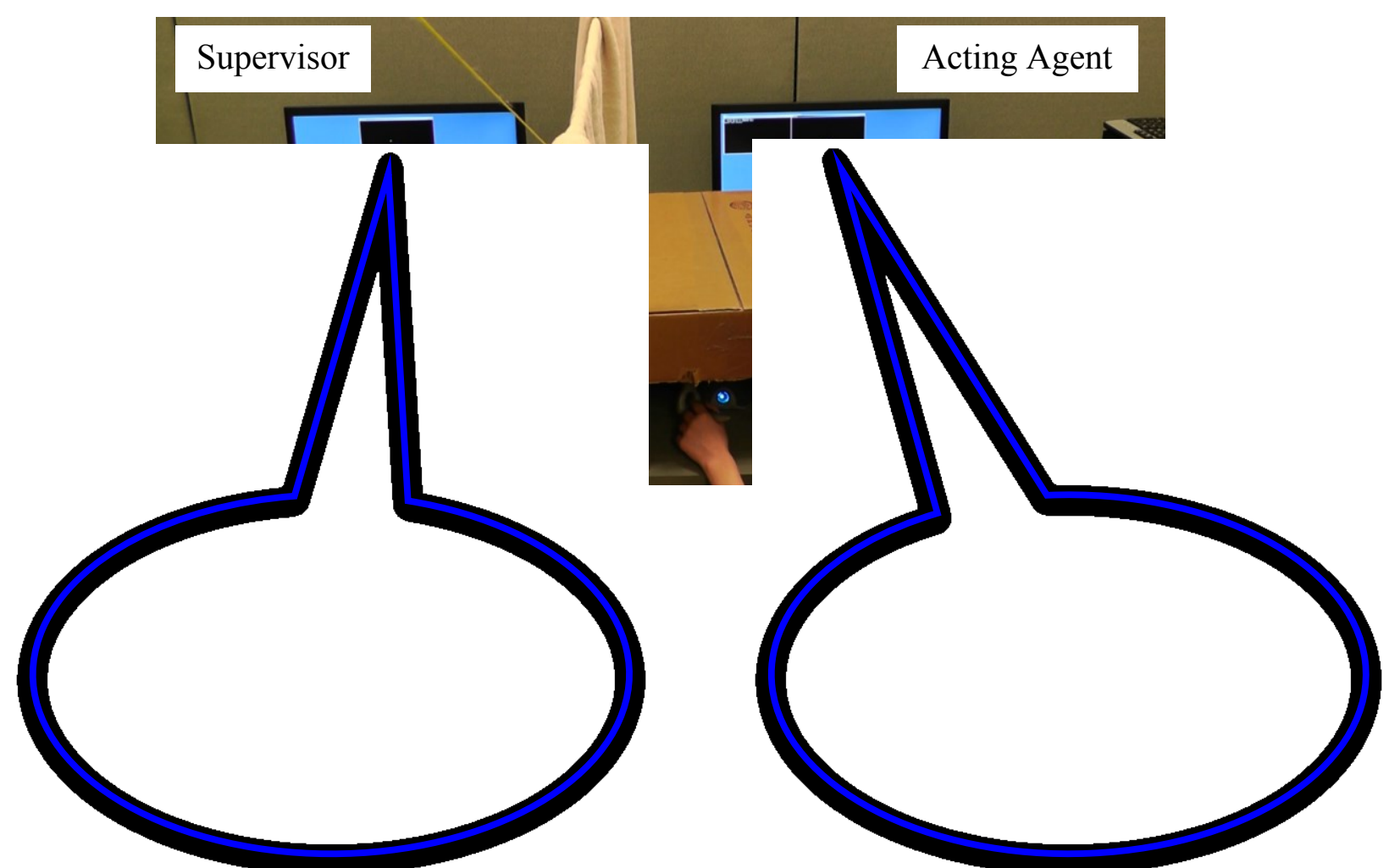

Figure 1. Experimental setup: the Supervisor could see the startıng point (yellow square, IU $\times I U$ pixels), the target (green square, $10 \times 10$ pixels) and the cursor (red square, $7 \times 7$ pixels) while the Acting Agent could see only the starting point and the cursor (red square and mouse cursor). The working window was $640 \times 480$ pixels.

\section{Experimental Design and Data Analysis}

This was a $6 \times 3$ mixed-design. The within-subject factor was the communication condition. It had three levels: haptic only $(\mathrm{H})$, verbal only (V), and the dual modality of haptic+verbal combined (HV). The between-subject factor was the order of communication conditions. It was fully counter-balanced, resulting in six orders. Three dyads were randomly assigned to each order. During the experiment, the trajectory of the mouse and haptic devices, task completion time, and verbalization were recorded for off-line analysis. The performance measures such as task completion time, path length, overshoot, and root mean square error (RMSE) were used to evaluate the effectiveness of the communication modality. Verbal data served to provide insight 
into possible difficulties or strategies in communication. A mixed-design ANOVA was used to analyze the data. An alpha level of .05 was used for all statistical tests.

\section{RESULTS}

\section{Task Completion Time}

The task completion time as a function of Index of Difficulty (ID) for the three communication conditions is shown in Figure 2. Modeled as a Fitts' Law task, the average task completion time fits a linear trend line (presented as $y=m x+b)$ for all three communication conditions $(\mathrm{H}, \mathrm{HV}$, and V) with a slope of $4.2(\mathrm{H}), 3.6(\mathrm{HV})$, and $3.5(\mathrm{~V})$, respectively. Participants' performance in the $\mathrm{V}$ and $\mathrm{HV}$ conditions were similar, while participants in the $\mathrm{H}$ only condition took $39.5 \%$ longer to complete the task. Additionally, performance in the $\mathrm{H}$ condition had a larger standard deviation.

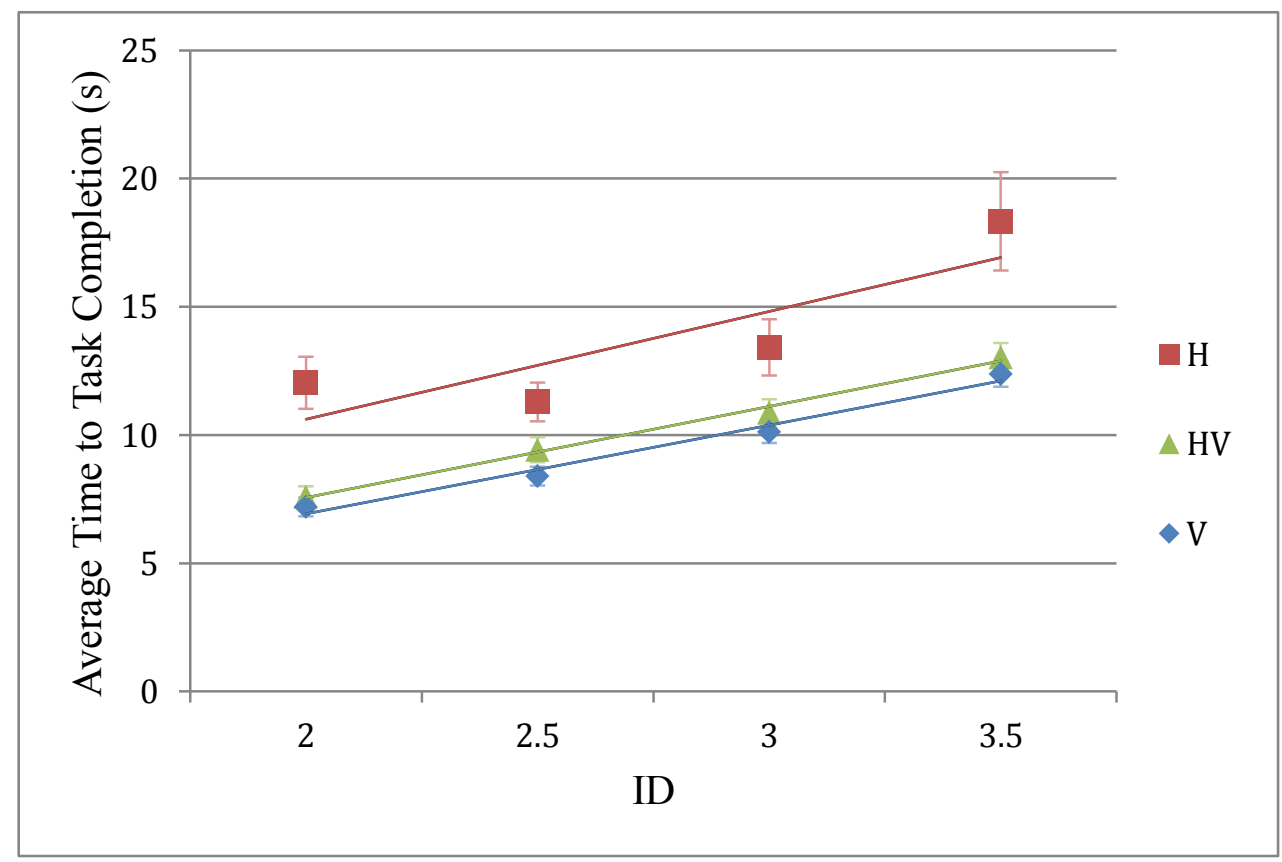

Figure 2. Mean and standard error of task completion time as a function of ID. 
The task completion time for the three communication conditions and the six counter-balanced orders are shown in Figure 3. ANOVA results showed that there was a significant main effect of order $\left(\mathrm{F}_{(5,12)}=3.34, p=.040, \mathrm{n}_{p}^{2}=.582\right)$, a significant main effect of communication condition $\left(\mathrm{F}_{(2}\right.$, 24) $\left.=19.66, p<.001, \eta_{p}^{2}=.621\right)$, and a significant interaction effect between order and communication condition $\left(\mathrm{F}_{(10,24)}=4.10, p=.002, \mathrm{\eta}_{p}^{2}=.631\right)$. Post-hoc Tukey HSD results showed that the difference between $\mathrm{H}-\mathrm{V}-\mathrm{HV}$ and $\mathrm{HV}-\mathrm{H}-\mathrm{V}$, was significant. Participants who began with haptics only (H-V-HV) performed significantly worse overall. Post-hoc Tukey HSD tests for communication condition indicated that the difference between $\mathrm{V}$ and $\mathrm{H}$, as well as between $\mathrm{H}$ and $\mathrm{HV}$, was significant. No significant difference between $\mathrm{V}$ and $\mathrm{HV}$ was found.

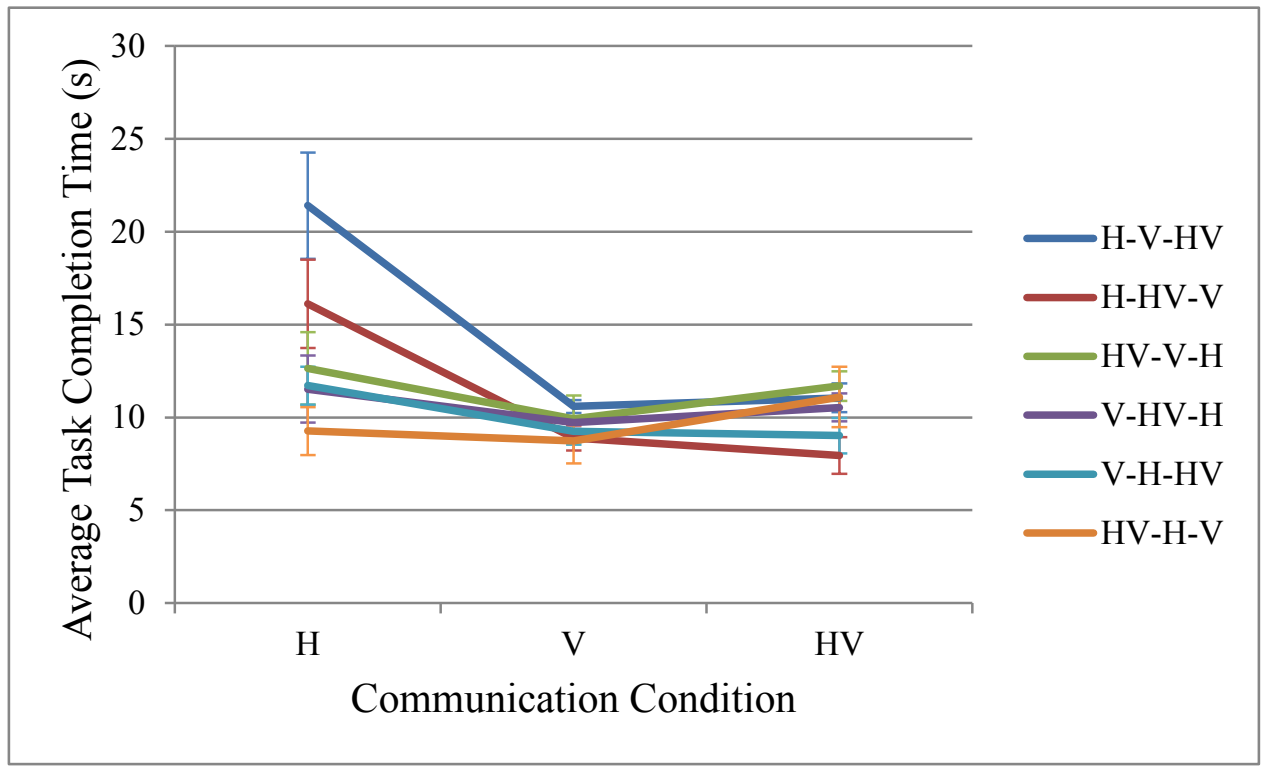

Figure 3. Mean and standard error of task completion time.

In further analysis, the task completion time was divided into three phases: movement initiation, travel, and termination (Figure 4). $\mathrm{T}_{1}$ denotes the time of the initiation phase which is the time period after receiving the Supervisor's instruction and ending with the movement of the cursor beyond the boundary of the starting position (the area around the starting position that is twice the length of the target, 20 pixels). $\mathrm{T}_{2}$ denotes the time spent travelling between starting point 
and target. This phase started immediately after the initiation phase and ended when the cursor entered the boundary of the target. It was the time spent moving in the general direction of the target without refining the final location. The time in the termination phase, $\mathrm{T}_{3}$, denotes the time spent within the boundary of the target (the area around the target that is twice the length of the target, 20 pixels). During this phase, the participants made adjustments to reach the final location and tended to give more specific instruction compared with the travel phase. $T_{4}$ is the sum of $T_{1}$ and $T_{2}$, and $T_{5}$ is the sum of $T_{2}$ and $T_{3}$. Therefore, the sum of $T_{1}, T_{2}$, and $T_{3}$ is the task completion time.

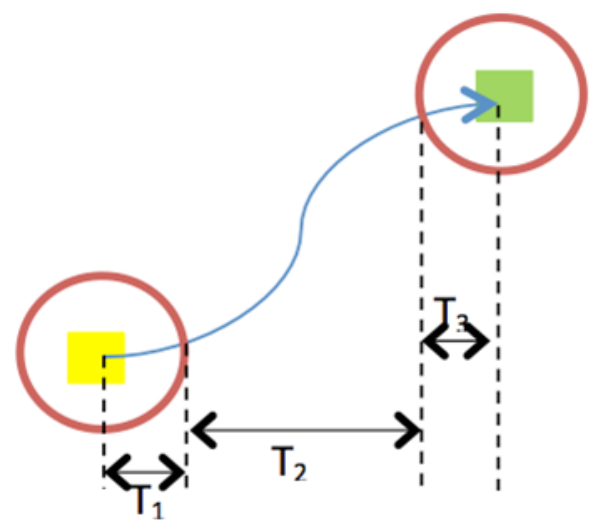

$$
\mathrm{T}_{4}=\mathrm{T}_{1}+\mathrm{T}_{2} ; \mathrm{T}_{5}=\mathrm{T}_{2}+\mathrm{T}_{3}
$$

Figure 4. Illustration of time phases in the movement. $T_{1}=$ movement initiation time, $T_{2}=$ navigation time, $\mathrm{T}_{3}=$ termination/refinement time. Total task completion time $=\mathrm{T}_{1}+\mathrm{T}_{2}+\mathrm{T}_{3}$.

Table 1 shows the mean value of $T_{1}$ to $T_{5}$ for each communication condition. For $T_{1}, T_{2}, T_{3}, T_{4}$, and $\mathrm{T}_{5}$, the statistical analysis results showed a significant difference between $\mathrm{V}$ and $\mathrm{H}$, and between $\mathrm{H}$ and $\mathrm{HV}$. No significant difference between $\mathrm{V}$ and HV was reported. It was observed that the condition of $\mathrm{H}$ only was the least efficient one for $\mathrm{T}_{2}, \mathrm{~T}_{3}, \mathrm{~T}_{4}$, and $\mathrm{T}_{5}$. Interestingly, the results for $\mathrm{T}_{1}$ showed that $\mathrm{H}$ only was the most efficient condition.

\section{Path Length, Overshoot, and Root Mean Square Error}


Because the distance between the starting position and the target location was different due to different IDs, relative path length was used for analysis. Relative path length was defined as the ratio of the actual path length of the mouse to the ideal shortest path length. The ANOVA results showed a main effect of order $\left(\mathrm{F}_{(5,12)}=8.67, p=.001, \mathrm{\eta}_{p}^{2}=.783\right)$ and communication condition $\left(\mathrm{F}_{(2,24)}=30.57, p<.001, \mathrm{n}_{p}^{2}=.718\right)$, as well as an interaction effect between order and communication condition $\left(\mathrm{F}_{(10,24)}=11.98, p<.001, \mathrm{\eta}_{p}^{2}=.833\right)$. For communication conditions, the post-hoc Tukey HSD test showed a significant difference between $\mathrm{V}$ and $\mathrm{H}$, as well as between $\mathrm{H}$ and $\mathrm{HV}$. There was no significant difference between $\mathrm{V}$ and $\mathrm{HV}$.

Finally, the overshoot of the mouse trajectory and root mean square error (RMSE) results also showed the same pattern (Table 2). The overshoot was defined as the distance between the target and a point on the mouse trajectory that was the furthest away from the starting point.

Table 1. Mean of time vs. communication condition. $\mathrm{T}_{1}$ : $\mathrm{H}$ was significantly shorter than $\mathrm{V}$ and $\mathrm{HV} ; \mathrm{V}$ and $\mathrm{HV}$ were not significant different. $\mathrm{T}_{2}-\mathrm{T}_{5}: \mathrm{H}$ was significantly longer than $\mathrm{V}$ and $\mathrm{HV} ; \mathrm{V}$ and $\mathrm{HV}$ were not significant different.

\begin{tabular}{lccccc}
\hline & $\mathrm{T}_{1}(\mathrm{~s})$ & $\mathrm{T}_{2}(\mathrm{~s})$ & $\mathrm{T}_{3}(\mathrm{~s})$ & $\mathrm{T}_{4}(\mathrm{~s})$ & $\mathrm{T}_{5}(\mathrm{~s})$ \\
\hline $\mathrm{H}$ & $1.76 \pm 0.54$ & $7.49 \pm 3.04$ & $4.52 \pm 3.16$ & $9.25 \pm 2.91$ & $12.01 \pm 5.28$ \\
$\mathrm{~V}$ & $2.03 \pm 0.44$ & $5.00 \pm 1.06$ & $2.49 \pm 0.77$ & $7.03 \pm 1.17$ & $7.49 \pm 1.26$ \\
$\mathrm{HV}$ & $2.21 \pm 0.68$ & $5.54 \pm 1.20$ & $2.47 \pm 0.84$ & $7.75 \pm 1.51$ & $8.01 \pm 1.71$ \\
\hline
\end{tabular}

Table 2. Summary of significant effects of communication conditions on the measures of time to task completion, relative path length, overshoot, and root mean square error. *Note: V\&H means that $\mathrm{V}$ and $\mathrm{H}$ are significantly different; $\mathrm{HV} \& \mathrm{H}$ means that $\mathrm{HV}$ and $\mathrm{H}$ are significantly different.

\begin{tabular}{|c|c|c|c|c|c|c|c|c|}
\hline \multirow[b]{3}{*}{ Measurement } & \multirow[b]{3}{*}{ Unit } & \multicolumn{3}{|c|}{$\mathrm{F}$ and $p$ value } & \multicolumn{3}{|c|}{ Mean \pm SD } & \multirow{3}{*}{$\begin{array}{l}\text { Tukey } \\
\text { HSD } \\
\text { test* }\end{array}$} \\
\hline & & Communication & & & & & & \\
\hline & & Condition & Order & Interaction & V & $\mathrm{H}$ & $\mathrm{HV}$ & \\
\hline $\begin{array}{l}\text { Time to task } \\
\text { completion }\end{array}$ & $\mathrm{s}$ & $\begin{array}{c}\mathrm{F}=19.66 \\
p<.001 \\
\mathrm{\eta}_{p}^{2}=.621\end{array}$ & $\begin{array}{l}\mathrm{F}=3.34, \\
p=.040, \\
\eta_{p}^{2}=.582\end{array}$ & $\begin{array}{c}\mathrm{F}=4.10 \\
p=.002 \\
\mathrm{\eta}_{p}^{2}=.631\end{array}$ & $\begin{array}{c}9.52 \\
\pm 1.39\end{array}$ & $\begin{array}{l}13.77 \\
\pm 5.00\end{array}$ & $\begin{array}{l}10.22 \\
\pm 2.01\end{array}$ & $\begin{array}{l}\text { V\&H; } \\
\text { HV\&H }\end{array}$ \\
\hline
\end{tabular}




\begin{tabular}{|c|c|c|c|c|c|c|c|c|}
\hline $\begin{array}{l}\text { Relative path } \\
\text { length }\end{array}$ & -- & $\begin{array}{c}\mathrm{F}=30.57 \\
p<.001 \\
\eta_{p}^{2}=.718\end{array}$ & $\begin{array}{l}\mathrm{F}=8.67, \\
p=.001, \\
\eta_{p}^{2}=.783\end{array}$ & $\begin{array}{c}\mathrm{F}=11.98, \\
p<.001, \\
\eta_{p}^{2}=.833\end{array}$ & $\begin{array}{c}2.32 \\
\pm 0.49\end{array}$ & $\begin{array}{c}5.75 \\
\pm 5.32\end{array}$ & $\begin{array}{c}2.42 \\
\pm 0.41\end{array}$ & $\begin{array}{l}\text { V\&H; } \\
\text { HV\&H }\end{array}$ \\
\hline Overshoot & pixel & $\begin{array}{c}\mathrm{F}=67.35 \\
p<.001 \\
\eta_{p}^{2}=.849\end{array}$ & $\begin{array}{l}\mathrm{F}=3.94, \\
p=.024, \\
\eta_{p}^{2}=.621\end{array}$ & $\begin{array}{l}\mathrm{F}=8.64 \\
p<.001 \\
\eta_{p}^{2}=.783\end{array}$ & $\begin{array}{l}13.41 \\
\pm 6.84\end{array}$ & $\begin{array}{c}37.14 \\
\pm 22.32\end{array}$ & $\begin{array}{l}16.77 \\
\pm 7.46\end{array}$ & $\begin{array}{l}\text { V\&H; } \\
\text { HV\&H }\end{array}$ \\
\hline $\begin{array}{l}\text { Root mean } \\
\text { square error }\end{array}$ & pixel & $\begin{array}{c}\mathrm{F}=81.91 \\
p<.001 \\
\eta_{p}^{2}=.872\end{array}$ & $\begin{array}{l}\mathrm{F}=4.85, \\
p=.012, \\
\mathrm{\eta}_{p}^{2}=.669\end{array}$ & $\begin{array}{l}\mathrm{F}=6.88, \\
p<.001, \\
\mathrm{\eta}_{p}^{2}=.741\end{array}$ & $\begin{array}{l}14.11 \\
\pm 3.66\end{array}$ & $\begin{array}{l}24.77 \\
\pm 8.67\end{array}$ & $\begin{array}{l}15.36 \\
\pm 3.14\end{array}$ & $\begin{array}{l}\text { V\&H; } \\
\text { HV\&H }\end{array}$ \\
\hline
\end{tabular}

\section{DISCUSSIONS}

\section{Performance of Haptic Modality}

The results based on task completion time, relative path length, overshoot, and RMSE were consistent and showed that Verbal communication, alone or combined with Haptics, was significantly better than Haptic communication only in this remote collaboration task. When using haptic feedback only, participants spent more time to complete the task, and had longer relative path length, larger overshoot, and bigger RMSE. Note that performance results for haptic modality also had a larger standard deviation. This indicates that the haptic modality, though sufficient, is less efficient than the verbal modality for this task. This may be due to the fact that participants were not familiar with using the haptic device and might have spent more time during trials to develop their communication strategies.

This is confirmed by the post-hoc observation that participants who went through the $\mathrm{H}$ communication condition after $\mathrm{V}$ and/or $\mathrm{HV}$ had better performance using the haptic modality than those participants who started with the $\mathrm{H}$ communication condition (orders of $\mathrm{H}-\mathrm{V}-\mathrm{HV}$ and $\mathrm{H}-\mathrm{HV}-\mathrm{V}$, Figure 3). The performance in the $\mathrm{V}$ and $\mathrm{HV}$ communication conditions were similar regardless of the order of the communication conditions. This means that haptic communication can be learned and using the verbal modality first is one way for users to learn to communicate through the haptic modality. 
If the initiation phase, $\mathrm{T}_{1}$, is an indication of reaction time, then the $\mathrm{H}$ modality was the most efficient communication channel for response initiation. This implies that the participants reacted quickly to the instruction given through the haptic modality. Haptic channel can be a good choice to provide warnings or alerts to elicit a quick response from participants.

\section{Relative Contribution of Haptic and Verbal Modalities}

Because there was no significant difference between $\mathrm{V}$ and $\mathrm{HV}$ conditions, it is not clear if the haptic modality was used when the verbal modality was also available. To verify, the ratio of the path length of the haptic device to the path length of the mouse in $\mathrm{H}$ and $\mathrm{HV}$ conditions were compared. If the Supervisor did not use the haptic modality to provide instruction, the motion of the haptic device driven by the Supervisor would not be related to the response of the Acting Agent as evidenced by the motion of the mouse. In that case, there would be a difference of the path length ratio between $\mathrm{H}$ and $\mathrm{HV}$ conditions. Analysis results showed that there was no significant difference $(p=.132)$ between the two conditions, indicating that the Supervisor did in fact use the haptic modality to communicate even when the verbal modality was available. There is no indication that haptic information was reduced by the Supervisor when the verbal modality was also used.

The post-hoc analysis of the motion range of the haptic device showed there was a difference between H and HV conditions $(p=.011)$. The Supervisor's haptic device in the HV condition had a smaller range of motion compared with the $\mathrm{H}$ condition. As the haptic instruction was provided by the Supervisor in both $\mathrm{H}$ and HV, it appears that the Supervisor exaggerated the motion of the haptic device in the absence of verbal communication.

Therefore, it was confirmed that the Supervisor gave commands using both Haptics and Verbalization in the HV condition, but it was unclear how the Acting Agent processed the 
information. It is possible that the Acting Agent relied only on verbalization in the verbal+haptic condition. It is also possible that this task was not difficult enough to require more than what could readily be provided and perceived through verbal commands.

\section{Communication Strategies}

In the conditions where $\mathrm{V}$ was available, we observed that verbal communication was dominated by the Supervisor issuing verbal commands even though the Acting Agent was allowed to talk. Participants were more likely to mimic the way their partner gave instructions in the first pairing before switching roles, but analysis showed that there was no difference on performance.

It was also noted that dyads employed two distinctly different strategies while performing the task. This was clearly reflected in the trajectory of the mouse movements. In the first strategy, the participants aimed the mouse in the general direction of the target and moved incrementally towards the target (Figure 5a). Analysis of the verbal instructions revealed that statements such as "move to northeast" were typical. This strategy was considered to be based on the Polar Coordinate System for navigation. In the second strategy, the task was achieved by following verbal instructions such as "move two inches to the right and then up", representative of a Cartesian Coordinate System (Figure 5b). The initial movement direction relied on the way the instruction was given, and was better aligned with the ideal path when the Polar Coordinate System was used to communicate.

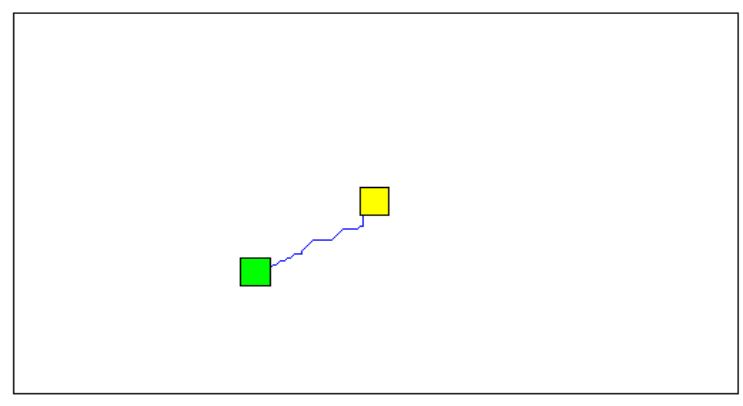

(a) Instruction with Polar Coordinate System

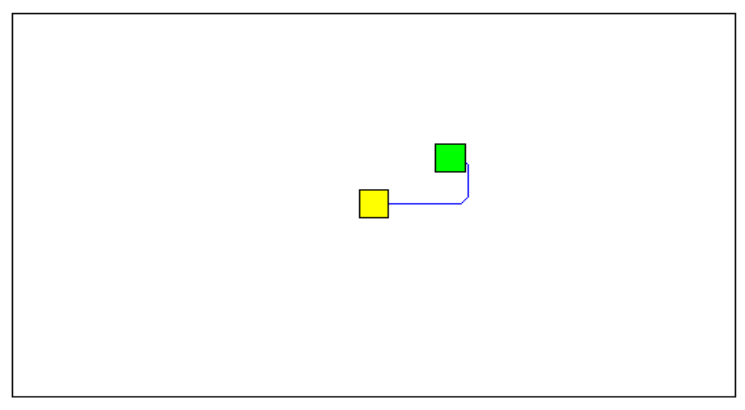

(b) Instruction with Cartesian Coordinate System 
Figure 5. Examples of mouse trajectory reflecting two different communication strategies.

It was also noted that the dyads tended to switch from the Polar Coordinate System to the Cartesian Coordinate System over the course of the experiment. A post-hoc analysis showed that this trend was statistically significant $(\mathrm{p}=.005)$ regardless of which communication condition or order was employed. To visualize this trend, a density gradient map was constructed to show the transition of communication strategy from Polar to Cartesian Coordinate System (Figure 6). In Figure 6(a), the high density area (darker grey) denotes a larger percentage of participants using the Polar coordinate system in each trial. The lighter grey denotes a smaller percentage of participants using the Polar coordinate system. Thus, over the course of the experiment (96 trials divided into 6 blocks for ease of comparison), the density map transitioned from dark gray to light gray, reflecting the trend towards favoring the Cartesian coordinate system with experience. Figure 6(b) shows the average results for each block. The darker color means more participants were using the Polar coordinate system for the targets in this block. Results suggest that the participants learned over the course of the experiment that it was more efficient to use the Cartesian system to share location information, regardless of the communication modality they were using. 
(a)

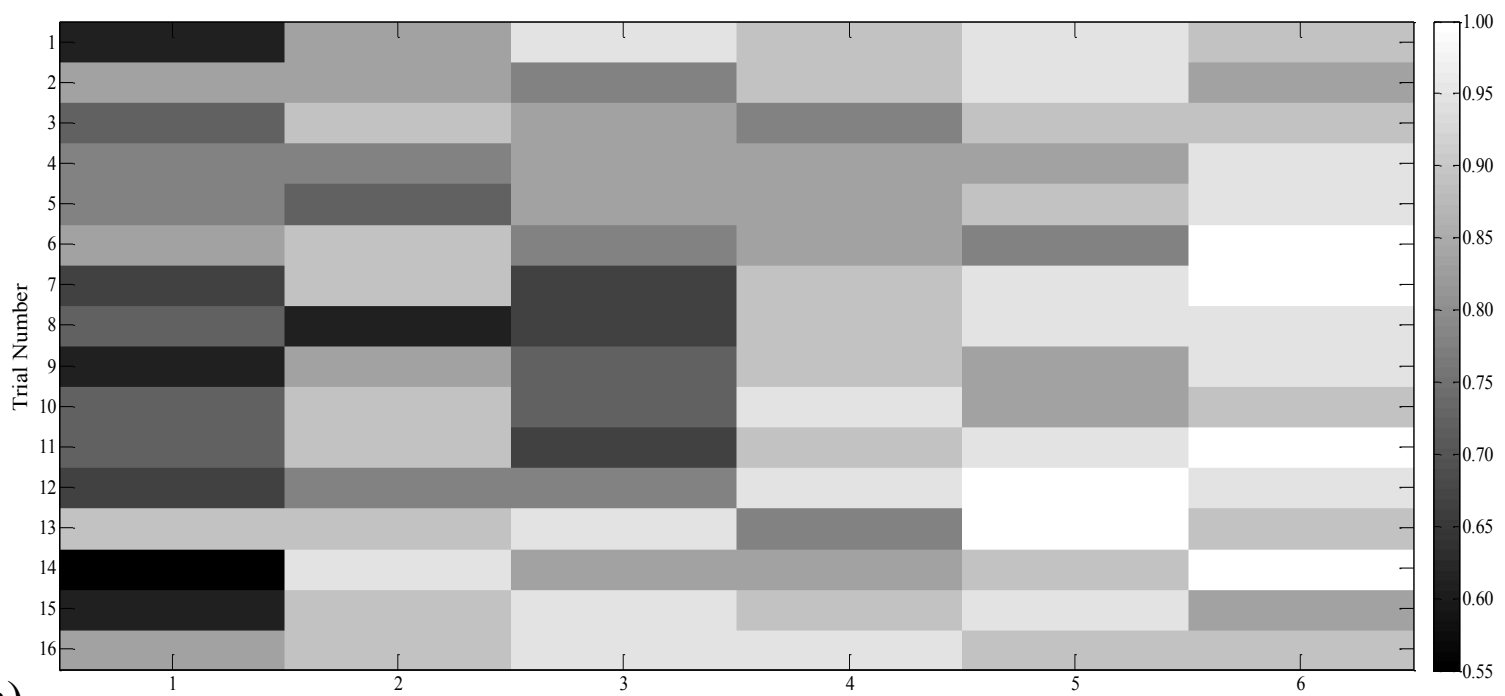

(b)

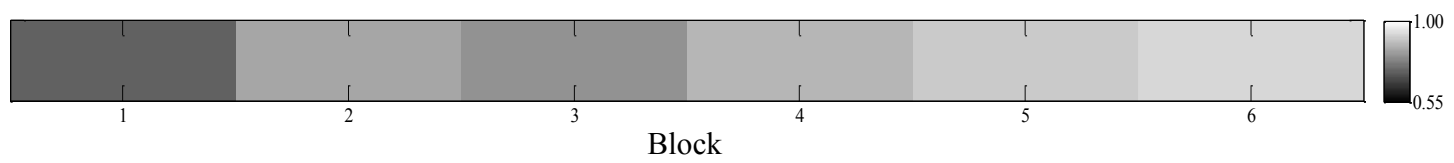

Figure 6. Results of classification by coordinate system. The grey scale represents the percentage of participants using the Cartesian system. The lighter the grey, the more participants used the Cartesian system in each trial or block. In (a), each cell represents one trial, from top to bottom ( $1^{\text {st }}$ trial to $16^{\text {th }}$ trial), and from left to right (each block representing 16 trials). In (b), each cell is an average of 16 trials. This schematic shows that participants converged on the use of the Cartesian coordinate system to communicate spatial information.

The idea that humans can hold different frames of reference (FOR) simultaneously (Lemay, Bertram, \& Stelamach, 2004) is not new. Different vocabularies are used for different FOR. For example, pilots use clock directions to describe locations when using the egocentric FOR during map-reading, but switch to using compass headings in a world-centered FOR when communicating with air traffic controllers (Aretz, 1991). The choice of which FOR to use during a collaborative task depends on the situation. Schober (1995) suggests that in situations where the people performing a task did not share the same point of view, the person giving instructions tended to give them from the other person's perspective. In our study, both Supervisor and Acting Agent had the same point of view of the task space, but described the task space using 
two different coordinate systems at the beginning of the experiment. This difference was likely due to the participants' varying backgrounds and "perceptual availability" of each coordinate system (Shepard \& Hurwitz, 1985). Engineering students likely have a greater "perceptual availability" or familiarity of the Polar coordinate system, while non-engineering students the Cartesian coordinate system. Therefore, the gradual transition to using mostly Cartesian coordinate system to communicate by the end of the experiment suggests that the dyads have established some common ground in this collaborative task.

\section{CONCLUSION}

The objective of this study was to investigate the influence of haptic communication during collaboration in a virtual environment. We hypothesized that haptics would improve performance in a collaborative pointing task. Results showed that although haptic feedback alone can be used to complete a collaborative virtual pointing task, it is less efficient than verbal feedback alone and verbal+haptic feedback combined.

\section{Limitations}

The current study is limited to a pointing task in a $2 \mathrm{D}$ virtual environment. The simplicity of the task removed the necessity to account for learning effects, but in fact may have been too easy a task to require more than verbal commands to accomplish collaboratively. In the experimental setup, only the trajectory of the master stylus and of the mouse were recorded, but not the timing of participants' verbalization. Therefore, the response time after verbal commands could not be determined. Consequently, the T1 measure includes both the initiation time and the time to move the cursor out of the boundary of the starting position.

As we are only beginning to understand how we might take advantage of haptic feedback to design for better collaborative work, we are still far from being able to convey useful information 
through haptic feedback only. Using haptics only to communicate is an unfamiliar experience to most hearing and speaking individuals. Therefore, we are not surprised that verbal communication dominates performance compared to using haptic only communication.

\section{Design Implications and Future Work}

Despite the limitations, our findings have several implications for the design of collaborative environments, as well as for real-world situations where guidance in navigation is done through visual or auditory queues (e.g, crane operators, navigation for the blind). The haptic modality can be very effective to communicate information in tasks requiring a quick response from a remote partner, thus can be used to send a warning or an alert. However, a training period may be necessary to establish a communication strategy, both in verbal communication and haptic communication. Ideally, the CVE can be designed to encourage the use of the appropriate coordinate system to communication spatial information, and support the use of the haptic channel to communicate temporal information or other critical parameters related to the task at hand. Future research will investigate the use of haptic communication in more complex collaborative tasks, as well as the use of haptics to convey information beyond physical properties.

\section{KEY POINTS}

- Haptics is an effective modality for communication, but is less efficient than verbal, in a collaborative virtual pointing task. Haptics is more efficient when used as a warning or an alert to elicit a quick response.

- The efficiency of haptic communication can be improved after a learning period, especially with the help of verbalization. 
- When both verbal and haptic modalities were available, participants used both modalities to give instruction. However, the instruction through the haptic channel was exaggerated when verbal modality was not available.

- With experience, participants were more likely to use the Cartesian coordinate system to communicate in the pointing task.

\section{REFERENCES}

Aretz, A.J. (1991). The design of electronic map displays. Human Factors, 33(1), 85-101.

Basdogan, C., Ho, C.-H., Srinivasan, M.A., \& Slater, M. (2000). An experimental study on the role of touch in shared virtual environments. ACM Transactions on Computer-Human Interaction (TOCHI), 7, 443-460.

Burdea, G.C. (1996). Force and touch feedback for virtual Reality. New York, NY, USA: John Wiley \& Sons, Inc.

Buxton, W. (1995). Touch, gesture \& marking. Human Computer Interaction: toward the Year 2000, Chapter 7, R.M. Baecker, J. Grudin, W. Buxton, \& S. Greenberg (Eds.). San Francisco: Morgan Kaufmann Publishers.

Chellali, A., Dumas, C., \& Milleville-Pennel, I. (2011). Influences of haptic communication on a shared manual task. Interacting with Computers, 23, 317-328.

Chellali, A., Dumas, C., \& Milleville-Pennel, I. (2012). Haptic communication to support biopsy procedures learning in virtual environments. Presence: Teleoperators and Virtual Environments, 21(4), 470-489.

Chellali, A., Milleville-Pennel, I., \& Dumas, C. (2013). Influence of contextual objects on spatial interactions and viewpoints sharing in virtual environments. Virtual Reality, 17 (1), 1-15. 
Churchill, E.F., \& Snowdon, D. (1998). Collaborative virtual environments: An introductory review of issues and systems. Virtual Reality, 3(1), 3-15.

Churchill, E. F., Snowdon, D. N., \& Munro, A. J. (2011). Collaborative Virtual Environments: Digital Places and Spaces for Interaction. (E. F. Churchill, D. N. Snowdon, \& A. J. Munro, Eds.) Springer Verlag.

Clark, H., \& Brennan, S. (1991). Grounding in communication. L. Resnick, J. Levine, \& S. Teasley (Eds.), Cognition, Perspectives on Socially Shared (pp. 127-149). Washington D.C.: American Psychological Association.

García, A.S., Molina, J.P., González, P., Martínez, D., and Martínez, J. (2009). An experimental study of collaborative interaction tasks supported by awareness and multimodal feedback. Proceedings of the 8th international conference on Virtual Reality Continuum and its Applications in Industry (VRCAI'09) (Yokohama, Japan, December 2009). pp. 77-82.

Gergle, D., Kraut, R.E., \& Fussell, S.R. (2012). Using visual information for grounding and awareness in collaborative tasks. Human Computer Interaction, 12(1), 1-39.

Gunn, C. (2006). Collaborative virtual sculpting with haptic feedback. Virtual Reality, 10, 73-83. Hanna, N., \& Richards, D. (2014). Evaluation framework for 3D collaborative virtual environments (THE CORE), PACIS2014 Proceedings. 284.

Harrison, S., \& Dourish, P. (1996). Re-place-ing space: the roles of place and space in collaborative systems. Proceedings of the 1996 ACM conference on Computer supported cooperative work (pp. 67-76). ACM.

Hubbold, R.J. (2002). Collaborative stretcher carrier: a case study. Proceedings of the 8th Eurographics Workshop on Virtual Environments (Barcelona, Spain, May 2002). pp. 7-12. 
Huang, Y.Y., Moll, J., Sallnäs, E.-L., \& Sundblad, Y. (2012). Auditory feedback in haptic collaborative interfaces. International Journal of Human-Computer Studies, 70(4), 257270.

Lecuyer, A. (2009). Simulating haptic feedback using vision: A survey of research and applications of pseudo-haptic feedback. Presence: Teleoperators and Virtual Environments, 18(1), 39-53.

LeMay, M., Bertram, C.P., Stelamach, G.E. (20014). Pointing to an allocentric and egocentric remembered target. Motor Control, 8, 16-32.

Lu, K., Liu, G., \& Liu, L. (2012). A study on haptic collaborative game in shared virtual environment. Fifth International Conference on Machine Vision (ICMV 2012): Computer Vision, Image Analysis and Processing, Proc. of SPIE, 8783, 87831Y-1 - 87831Y-7.

MacKenzie, I.S. (1992). Fitts' law as a research and design tool in human-computer interaction. Human-Compter Interaction, 7, 91-139.

Moll, J., Huang, Y., \& Sallnas, E.-L. (2010). Audio makes a difference in haptic collaborative virtual environments. Interacting with Computers, 22(6), 544-555.

Reed, K.B., \& Peshkin, M.A. (2008). Physical collaboration of human-human and human-robot teams. IEEE Transactions on Haptics, 1(2), 108-120.

Schober, M.F. (1995). Speakers, addresses, and frames of reference: Whose effort is minimized in conversations about locations? Discourse Processes, 20, 219-47.

Shepard, R., Hurwitz, S. (1985). Upward direction, mental rotation, and discrimination of left and right turns in maps. Visual Cognition, Steven Pinker, Ed. MIT Press, pp. 161-93.

Takac, B., Chellali, A., Dumas, C., Milleville-Pennel, I., Grosdemouge, C., \& Cao, C.G.L. (2011). Haptic communication for a 2D pointing task in a virtual environment. 
Proceedings of the Human Factors and Ergonomics Society Annual Meeting (HFES 2011), $55,2168-2172$.

Ullah, S. (2011). Multi-modal assistance for collaborative 3D interaction: Study and analysis of performance in collaborative work. Human-Computer Interaction. Université d' EvryVal d' Essonne.

Ullah, S., Liu, X., Otmane, S. Richard, P., \& Mallem, M. (2011). What you feel is what I do: A study of dynamic haptic interaction in distributed collaborative virtual environment. Human-Computer Interaction, Part II (HCII 2011), J.A. Jacko (Ed.). Berlin, Heidelberg: Springer-Verlag. 140-147.

Wang, J., Chellali, A., \& Cao, C.G.L. (2013). A study of communication modalities in a virtual collaborative task. IEEE International Conference on Systems, Man, and Cybernetics (SMC2013), 542-546.

Wright, T.E., \& Madey, G. (2008). A survey of collaborative virtual environment technologies. Tech. rep., University of Notre Dame-USA.

Young, S., Moon, H.-Y., Sohn, Y., \& Fernandes, M. (2008). A survey of security issue in collaborative virtual environment. IJCSNS International Journal of Computer Science and Network Security, 8(1), 14-19.

\section{BIOGRAPHIES}

Jinling Wang is currently a postdoctoral research associate in the Ergonomics in Remote Environments Laboratory at Wright State University. She received her Ph.D. in Mechanical Engineering from the National University of Singapore in 2011 and a Bachelor degree in Automation from the University of Science and Technology of China in 2006. 
Amine Chellali is an Assistant Professor in the Department of computer engineering at the University of Evry-Val-d'Essonne (France), conducting his research within the IRA2 team at the IBISC Laboratory. He received his $\mathrm{PhD}$ in Human Computer Interaction from the University of Nantes (France) in 2009. Previously, he worked as a research assistant at Ecole des Mines de Nantes. He also worked as a postdoctoral associate at Tufts University in the Ergonomics in Remote Environments Laboratory and then as a research associate in the Interactive Surgical Systems Laboratory at Harvard Medical School. His research interests are in the fields of virtual reality and human computer interaction. He is more particularly interested in Task-Centered design, Haptic interaction, Surgical training systems and Communication in Collaborative Virtual Environments (CVE)

Caroline G. L. Cao received a Ph.D. in mechanical \& industrial engineering from the University of Toronto in 2002. She is currently Professor of Biomedical, Industrial and Human Factors Engineering and Director of the Ergonomics in Remote Environments Laboratory (EREL) at Wright State University. She also carries the title of Ohio Research Scholar with the Ohio Imaging Research and Innovation Network (OIRAIN). Professor Cao's research is in the area of human factors of medical systems, including the design and evaluation of enabling technology (e.g., robotics, image guidance, haptics) for minimally invasive surgery, training of surgical skills in training simulators, and decision-making and team communication in the operating room. Dr. Cao is a recipient of the National Science Foundation Career Award. She was a visiting professor at the Ecole des Mines de Nantes, and a visiting researcher in the Robotics Team and the PsyCoTec Team at the IRCCyN (Institut de Recherche en Communications et en Cybernetique de Nantes), with a Chaires Regionales de Chercheurs Etrangers award from the Research Council of la Region des Pays de la Loire in 2009-2011. She was also a Distinguished 
Visiting Scientist at the Australian E-Health Research Centre at CSIRO (Commonwealth Scientific and Industrial Research Organisation) in Brisbane, Australia in 2013. 